\title{
Publisher's Note: Metal nanospheres under intense continuous-wave illumination: A unique case of nonperturbative nonlinear nanophotonics \\ [Phys. Rev. E 96, 012212 (2017)]
}

I. Gurwich and Y. Sivan

(Received 7 November 2017; published 13 November 2017)

DOI: 10.1103/PhysRevE.96.059901

This paper was published on 14 July 2017 with errors in Figures 3 and 4 and the caption to Fig. 3 . Figures 3 and 4 have been replaced. The caption to Fig. 3 should read as "Same as Fig. 1 for a Ag nanosphere (permittivity data taken from Ref. [24]) and $\epsilon_{h, 0}^{\prime}=6.15 . "$

The figures and caption noted above have been corrected as of 7 November 2017. The figures and caption are incorrect in the printed journal. For the benefit of the print readership, the corrected figures are duplicated below.

The authors are grateful to Ieng-Wai Un for bringing these errors to their attention.
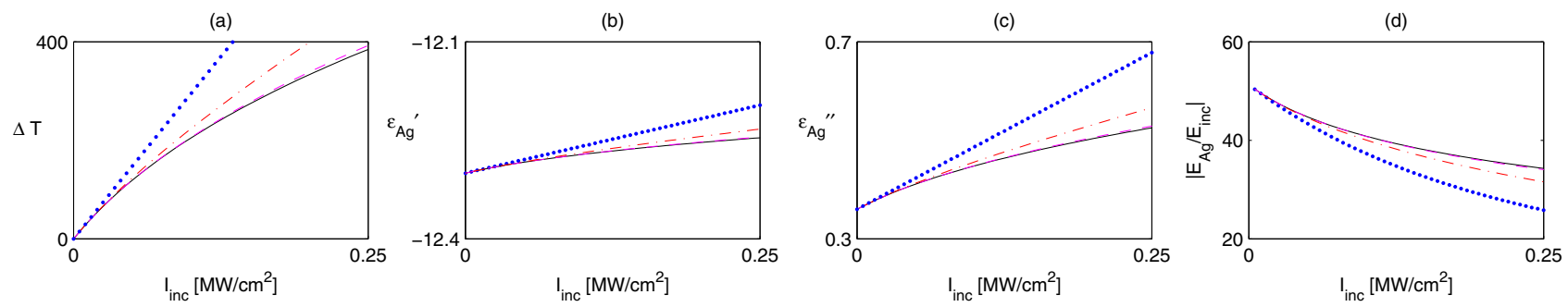

FIG. 3. Same as Fig. 1 for a Ag nanosphere (permittivity data taken from Ref. [24]) and $\epsilon_{h, 0}^{\prime}=6.15$.
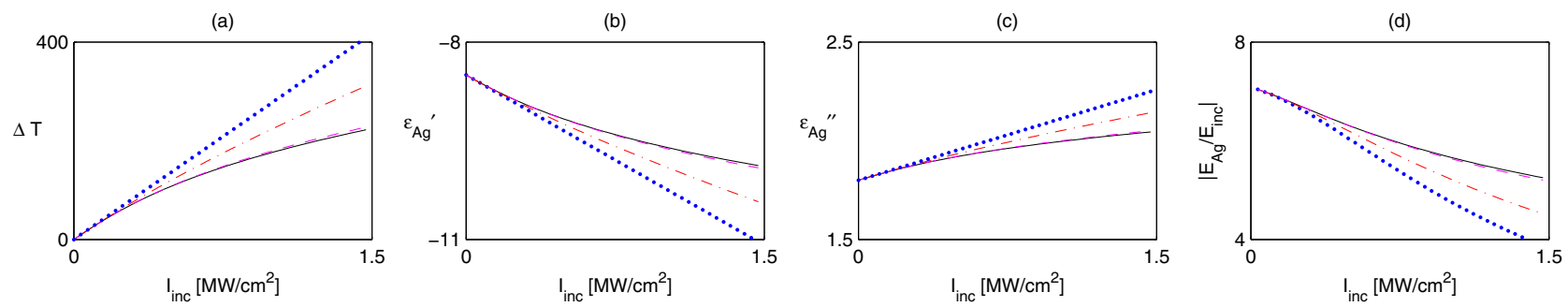

FIG. 4. Same as Fig. 3 but with the permittivity data taken from Ref. [50], hence, with $\epsilon_{h, 0}^{\prime} \sim 4$. 